Submitted to the Astrophysical Journal Letters

Preprint typeset using $\mathrm{AT}_{\mathrm{E}} \mathrm{X}$ style emulateapj v. 5/2/11

\title{
EVIDENCE THAT GAMMA-RAY BURST 130702A EXPLODED IN A DWARF SATELLITE OF A MASSIVE GALAXY
}

\author{
Patrick L. Kelly, Alexei V. Filippenko, Ori D. Fox, Weikang Zheng, and Kelsey I. Clubb \\ Department of Astronomy, University of California, Berkeley, CA 94720-3411, USA \\ Submitted to the Astrophysical Journal Letters
}

\begin{abstract}
GRB 130702A is a nearby long-duration gamma-ray burst (LGRB) discovered by the Fermi satellite whose associated afterglow was detected by the Palomar Transient Factory. Subsequent photometric and spectroscopic monitoring has identified a coincident broad-lined Type Ic supernova (SN), and nebular emission detected near the explosion site is consistent with a redshift of $z=0.145$. The SNGRB exploded at an offset of $\sim 7.6^{\prime \prime}$ from the center of an inclined $r=18.1$ mag red disk-dominated galaxy, and $\sim 0.6^{\prime \prime}$ from the center of a much fainter $r=23$ mag object. We obtained Keck-II DEIMOS spectra of the two objects and find a $2 \sigma$ upper limit on their line-of-sight velocity offset of $\lesssim 60 \mathrm{~km} \mathrm{~s}^{-1}$. If we calculate the inclination angle of the massive red galaxy from its axis ratio and assume that its light is dominated by a very thin disk, the explosion would have a $\sim 60 \mathrm{kpc}$ central offset, or $\sim 9$ times the galaxy's half-light radius. A significant bulge or a thicker disk would imply a higher inclination angle and greater central offset. The substantial offset suggests that the faint source is a separate, dwarf galaxy. The star-formation rate of the dwarf galaxy is $\sim 0.05 \mathrm{M}_{\odot} \mathrm{yr}^{-1}$, and we place an upper limit on its oxygen abundance of $12+\log (\mathrm{O} / \mathrm{H})<8.16$ dex. The identification of an LGRB in a dwarf satellite of a massive, metal-rich primary galaxy suggests that recent detections of LGRBs spatially coincident with metal-rich galaxies may be, in some cases, superpositions.

Subject headings: gamma-ray burst: individual (GRB 130702A) - supernovae: general — galaxies: star formation - galaxies: abundances
\end{abstract}

\section{INTRODUCTION}

The high-energy photons and power-law afterglow characteristic of long-duration gamma-ray bursts (LGRBs), as well as the existence of coincident broadlined Type Ic supernovae (SNe Ic-BL; Galama et al. 1998; Matheson et al. 2003; Stanek et al. 2003; Hiorth et al. 2003), are consistent with the creation of a relativistic jet after the core of a massive star collapses to form a compact object (Woosley et al. 1993). Theoretical modeling of possible progenitor systems strongly favors massive, rapidly spinning stars (Hirschi et al. 2005; Yoon \& Langer 2005; Wooslev \& Heger 2006), because high angular momentum is likely required to form the relativistic jets. These massive stars must also lose their outer hydrogen and helium envelopes, or burn them through internal mixing (Yoon \& Langer 2005; Woosley \& Heger 2006), because all associated SNe have had Type Ic-BL spectra which show no evidence for hydrogen or helium.

The metal content of LGRB host galaxies has received special attention because of the strong effect of metals on stellar mass loss rates. The outflowing stellar winds from massive stars grow in strength with increasing metallicity $(Z)$ of the envelope (Kudritzki \& Puls 2000) approximately as $\dot{M} \propto Z^{0.66}$ (Vink \& de Koter 2005). Single Wolf-Rayet stars with low metallicity are expected to have higher rates of rotation than their high-metallicity counterparts, and a reasonable expectation might be that LGRBs would occur more frequently in metal-poor environments, where single stars may retain more of their angular momentum before core collapse. However, re-

pkelly@astro.berkeley.edu cent observations have suggested that many massive stars have binary companions (e.g., Sana et al. 2012). Interaction with a close companion may be able to produce stars with the high angular momentum and stripped envelopes necessary for an LGRB (Zhang \& Fryer 2001; Fryer \& Heger 2005), even in comparatively metal-rich environments.

Modiaz et al. (2008) found that SNe Ic-BL with an LGRB strongly prefer more metal-poor hosts than those without a coincident LGRB (see also Stanek et al. 2006; Graham \& Fruchter 2012). Recent host-galaxy follow-up observations, however, have found examples of LGRBs that exploded at positions coincident with metal-rich host galaxies (e.g., Prochaska et al. 2007; Levesque et al. 2010; Savaglio et al. 2012; see, among others, Kocevski \& West 2011 for possible interpretations).

Here we show an example of an LGRB that probably exploded in a metal-poor dwarf satellite of a metalrich galaxy. A plausible possibility is that a fraction of the LGRBs whose positions have coincided with metalrich galaxies may have less luminous, metal-poor host galaxies that are difficult to detect. The low redshift $(z=0.145)$ of GRB 130702A allows the separation of the dwarf neighbor host from a massive primary galaxy in ground-based images, and makes possible the spectroscopic detection of weak emission lines from the dwarf galaxy's metal-poor gas. Section 2 briefly discusses the discoveries and multi-wavelength observations of the GRB, coincident optical transient, and associated SN Ic-BL by several observing teams. Section 3 describes photometry and spectroscopy of the sources close to the SN-GRB site. We present results from our photometric and spectroscopic analyses in 4 , and we provide both a 
summary and interpretation in $\$ 5$.

\section{DETECTION AND FOLLOW-UP OBSERVATIONS}

GRB $130702 \mathrm{~A}$ was detected by the Fermi-GBM (Collazzi et al. 2013) and Fermi-LAT (Cheung et al. 2013) on 2 July 2013 (UT dates are used throughout this paper). Analysis of the Fermi Gamma-ray Burst Monitor (GBM) light curve showed a burst duration of $T_{90} \approx 59 \mathrm{~s}(50-300 \mathrm{keV}$; Collazzi et al. 2013). The Palomar Transient Factory (PTF) identified an optical afterglow at coordinates $\alpha=14^{\mathrm{h}} 29^{\mathrm{m}} 14.78^{\mathrm{s}}, \delta=+15^{\circ} 46^{\prime} 26.4^{\prime \prime}$ (J2000.0) at a distance of $3.8^{\circ}$ from the center of the Fermi-GBM trigger 394416326 localization (Singer et al. 2013). Follow-up observations of the optical transient designated as iPTF13bxl by Singer et al. (2013) yielded an $r^{\prime}=18.7 \mathrm{mag}$ estimate at $4: 10$ on 2013 July 3 . These authors also noted the existence of a nearby faint $r=23.01 \mathrm{mag}$ source SDSS J142914.75+154626.0 separated by $0.6^{\prime \prime}$ from the optical transient, and a bright red $(r \approx 18 \mathrm{mag} ; g-r \approx 0.9 \mathrm{mag})$ galaxy SDSS J142914.57+154619.3, at a separation of $7.6^{\prime \prime}$.

Butler et al. (2013a) reported optical and infrared measurements of the optical transient with the RATIR telescope, while CARMA (Perley et al. 2013) observations detected the transient at millimeter wavelengths. RATIR monitoring showed a flattening of the afterglow light curve on 6 and 7 July 2013, five days after the Fermi detection (Butler et al. 2013b). A spectrum taken by Schulze et al. (2013) on 8 July 2013 of the optical afterglow shows evidence for broad features, and Cenko et al. (2013) and D'Elia et al. (2013) obtained additional spectra that suggested that the coincident Type Ic-BL SN was similar to SN 1998bw and SN 2006aj.

Mulchaey et al. (2013) reported weak $\mathrm{H} \alpha$ and [O III] emission lines superimposed on the spectrum of the optical afterglow indicative of $z=0.145$. A spectrum taken by Leloudas et al. (2013) of the bright red galaxy offset by $7.6^{\prime \prime}$ from the optical transient showed weak $\mathrm{H} \alpha$ and [O II] emission lines, but these authors did not detect emission lines at the explosion site.

\section{DATA AND ANALYSIS}

The $2.5 \mathrm{~m}$ telescope at Apache Point, New Mexico was used to perform the Sloan Digital Sky Survey (SDSS) imaging and spectroscopic surveys. The imaging component, consisting of $53.9 \mathrm{~s}$ integrations through the SDSS ugriz filter set, spans 14555 square degrees through Data Release 9 (DR 9; Ahn et al. 2012), and has an average magnitude limit (95\% point-source completeness) of $r \approx 22.2 \mathrm{mag}$. Each SDSS image has a $13.5^{\prime} \times 9.9^{\prime}$ format recorded by a $2048 \times 1498$ pixel array. We correct all galaxy magnitudes for extinction by the Galactic dust along the line of sight using the Schlafly \& Finkbeiner (2011) recalibration of the Schlegel et al. (1998) map.

With the LePhare code (Arnouts et al. 1999), we fit SDSS ugriz galaxy magnitudes with spectral energy distributions (SEDs) from the PEGASE2 (1999) stellar population synthesis models. The SEDs include both nebular and stellar light and track metal enrichment and metal-dependent extinction through 69 timesteps for each of the size models. We use the initial mass function (IMF) of Rana \& Basu (1992) with a 5\% fraction of close binaries and a mass range of 0.09 to $120 \mathrm{M}_{\odot}$.
We acquired two $600 \mathrm{~s}$ Keck/DEIMOS (Faber et al. 2003 ) spectra of the optical afterglow and adjacent faint source with the $600 \mathrm{l} \mathrm{mm}^{-1}$ grating and $1^{\prime \prime}$ slit (full width at half-maximum intensity $[\mathrm{FWHM}]$ resolution $\sim 3.5 \AA$ ) in clear conditions on 11 July 2013. The spectra were taken with a slit position angle (PA) of $50.4^{\circ}$ which allowed it to intersect both the optical transient and the faint adjacent SDSS source that we identify as the dwarf LGRB host galaxy. The data were taken at an airmass of 1.2 , and the seeing was $\sim 1^{\prime \prime}$ during the observations.

Immediately following the above pair of integrations, we obtained a $60 \mathrm{~s}$ DEIMOS spectrum with the slit positioned on the center of the bright red galaxy. The purpose of this integration, also taken with a PA of $50.4^{\circ}$, was to be able to compare the line-of-sight velocities of the faint source and the bright red galaxy.

All spectra were reduced using IRAF routines and the two-dimensional subtraction techniques developed by Kelson (2003). The spectra were flux calibrated using the $\mathrm{BD}+26^{\circ} 2606$ spectrophotometric standard star (Oke \& Gunn 1983), and corrections were made for telluric atmospheric absorption features. We extracted line strengths after flux calibration by fitting a Gaussian to each line profile. The uncertainty of each line-flux estimate was computed using the method described by Pérez-Montero \& Díaz (2003). Figure 1 displays image sections of the background- and sky-subtracted spectra centered around the $\mathrm{H} \alpha$ emission lines.

\section{RESULTS}

Figure2 shows the field surrounding the coordinates of the optical counterpart of GRB 130702A, and the label that we assign to each object. The faint source S1 closest to the center of the crosshairs is the likely host of the LGRB. We find evidence that S1 is bound to or interacting with the bright red disk-dominated galaxy (labeled S2) having an offset of $7.6^{\prime \prime}(\sim 19.1 \mathrm{kpc}$ in projection) from the GRB coordinates. Source S3 is a bright blue galaxy $15.8^{\prime \prime}(\sim 37.8 \mathrm{kpc}$ in projection) southeast of the GRB. We have no spectroscopic redshift for S3, but its SDSS photometric redshift is $0.19 \pm 0.11$, consistent with the 0.145 spectroscopic redshift of S1 and S2. A large number of faint sources with close proximity to S1 suggest the possibility of local-galaxy interaction.

For the dwarf host galaxy $\mathrm{S} 1$, we measure an $\mathrm{H} \alpha$ wavelength of $7514.4 \pm 0.3$ (statistical) $\AA$ using a Gaussian fit to the line profile. A fit to the broader $\mathrm{H} \alpha$ emission profile extracted from an aperture including the central region of the red bright galaxy $\mathrm{S} 2$ yields an $\mathrm{H} \alpha$ wavelength of $7515.4 \pm 0.7$ (statistical) $\AA$. These are consistent with $z \approx 0.145$ measured by Mulchaey et al. (2013) from emission lines superimposed on the afterglow spectrum, and are approximately consistent with the redshift expected for the SN Ic-BL associated with GRB 130702A based on the observer-frame wavelengths of its broad features reported by Schulze et al. (2013). We use Monte Carlo simulations to compute the uncertainty of the wavelength measurements. We estimate that these DEIMOS observations place a $2 \sigma$ upper limit on the velocity difference between the $\mathrm{S} 1$ and $\mathrm{S} 2$ galaxies of $\sim 60 \mathrm{~km} \mathrm{~s}^{-1}$.

From fitting PEGASE2 stellar population synthesis models to the SDSS ugriz broadband photometry, we estimate the stellar masses of the faint LGRB 

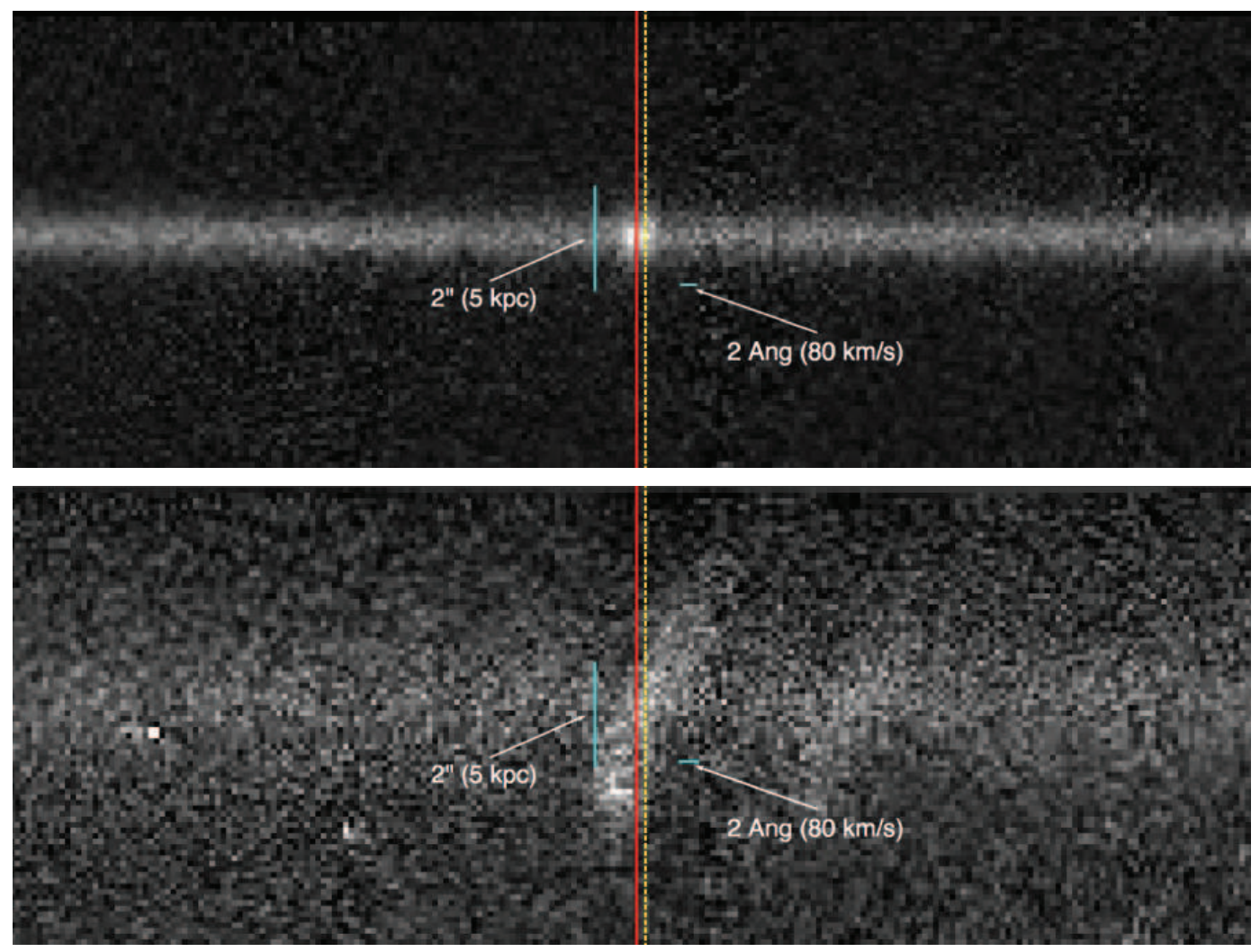

Figure 1. Background- and sky-subtracted Keck spectra of the optical afterglow of GRB 130702A and faint adjacent source S1 (upper panel), and of the bright red galaxy S2 (lower panel). The abscissa corresponds to the dispersion axis, while the ordinate is the spatial axis along the $1^{\prime \prime}$ slit. Each panel shows the trace and superimposed $\mathrm{H} \alpha$ emission line (as well as [N II] in the bottom panel). The red solid line marks the $7514.4 \pm 0.3$ (statistical) $\AA$ central wavelength we measure for the $\mathrm{H} \alpha$ emission line of the dwarf host galaxy S1, and the yellow dashed line marks the 7515.4 \pm 0.7 (statistical) $\AA$ wavelength of the emission line of the bright red galaxy S2. The upper panel demonstrates that the $\mathrm{H} \alpha$ emission from $\mathrm{S} 1$ has the same position along the spatial axis as the continuum light from the optical afterglow, suggesting an association between the LGRB and S1. The wavelength profile of the emission lines in the lower panel reflects the rotation of the galaxy's disk.

host galaxy $\mathrm{S} 1\left(10^{7.9 \pm 0.7} \mathrm{M}_{\odot}\right)$, the bright red galaxy S2 $\left(10^{10.8 \pm 0.1} \mathrm{M}_{\odot}\right)$, and the blue nearby galaxy S3 $\left(10^{9.5 \pm 0.08} \mathrm{M}_{\odot}\right)$. This suggests that the massive red galaxy $\mathrm{S} 2$ dominates the local gravitational potential, even if the blue galaxy S3 has close physical proximity. Table 1 lists the offsets from GRB 130702A, stellar masses, and abundance limits that we estimate for these three galaxies.

The SDSS photometry pipeline performs separate fits of a de Vaucouleurs profile and of an exponential profile to the light distribution of each galaxy. While holding all parameters from these separate fits constant except the profiles' amplitudes, the pipeline next finds the linear combination of the two profiles that has the best agreement with flux distribution. The logarithmic likelihood of a de Vaucouleurs profile fit to the $r$-band light of the bright red galaxy S2 is -217.3, exceedingly small, while the logarithmic likelihood of an exponential profile is -3.4 . In the combined fit, the exponential profile receives $98.4 \%$ of the flux. Using the scale length of the SDSS exponential profile, we compute that the massive red galaxy $\mathrm{S} 2$ has a half-light radius of $\sim 10.9 \mathrm{kpc}$. Our spectrum of $\mathrm{S} 2$ shown in Figure 1 at $\mathrm{PA}=50.4^{\circ}$ exhibits evidence for coherent rotation in a disk galaxy with ongoing star formation.

We use the "expAB_r" SDSS pipeline estimate of the ratio $b / a$ (semiminor to semimajor axis lengths) of the massive red galaxy S2. If we assume that a very thin disk dominates the galaxy's light (i.e., the disk's thickness does not contribute significantly to the size of the projected semiminor axis), the best-fitting value of $b / a=0.22 \pm 0.06$ corresponds to an inclination angle of $\sim 77 \pm 4^{\circ}$. The disk thickness or the bulge likely makes a significant contribution to the apparent semiminor axis length, so the disk is probably even more inclined. The SDSS exponential fit to the $r$-band galaxy flux also finds that the angle of the semimajor axis of the galaxy is $67^{\circ}$ counterclockwise north to east. If we make the assumptions that the GRB explosion site is in the plane of the galaxy and that the disk is very thin, then projection of the GRB coordinates onto the plane of the disk yields an offset from the galaxy center of $\sim 60 \mathrm{kpc}$. This distance is $\sim 9$ times the half-light radius. Given the possibility that the disk thickness is significant or that the bulge is substantial, the LGRB offset may be much greater than this value.

The SDSS spectroscopic galaxy survey targeted objects detected by the imaging survey with $5 \sigma$ significance, an extended profile, and an $r^{\prime}$-band magnitude brighter than 17.77. Within $15^{\prime}(\sim 2.3 \mathrm{Mpc}$ in projection) of the GRB coordinates, five bright galaxies have spectroscopic redshifts (0.1427921, 0.1447673, 0.1411451, 0.1418472, $0.1423725)$ which are similar to the 0.145 redshift of the dwarf LGRB host galaxy and red massive companion. 


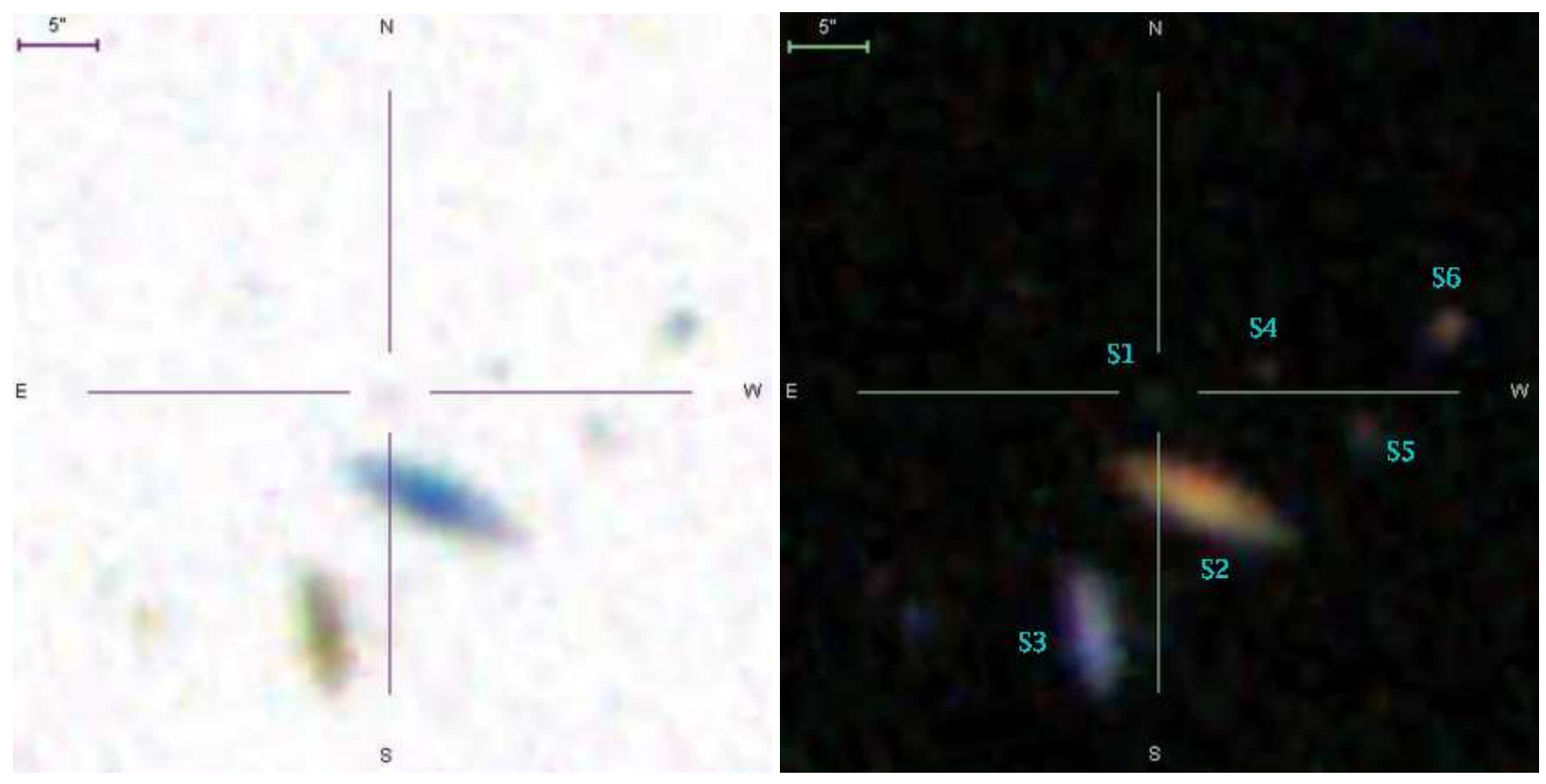

Figure 2. SDSS color composite (left panel) and colormap-inverted (right panel) images of the field surrounding GRB 130702A. The position of the LGRB optical afterglow is marked with crosshairs. The center of a faint $r=23.01$ mag source $\mathrm{S} 1$ is $\sim 0.6^{\prime \prime}$ (projected $\sim 1.5 \mathrm{kpc}$ ) from the GRB position, while a bright $r=18.14$ mag galaxy S2 is 7.6" (projected $\sim 19.1 \mathrm{kpc}$ ) from the GRB. The bright blue galaxy S3 $(r=19.02 \mathrm{mag})$ has an offset of $15.8^{\prime \prime}(37.8 \mathrm{kpc})$ and a photometric redshift $0.19 \pm 0.11$. The source S4 $(r=22.91 \mathrm{mag})$ west of PTF13bxl with offset $7.5^{\prime \prime}$ (18.4 kpc in projection) is unresolved in SDSS images and has no available photometric redshift. The red source S5 $(r=21.42 \mathrm{mag})$ west of PTF13bxl with offset $14.4^{\prime \prime}(35.6 \mathrm{kpc}$ in projection) has a photometric redshift $0.17 \pm 0.10$, also consistent with the S1 and S2 redshifts. The red galaxy S6 farther to the north and west with offset $19.1^{\prime \prime}$ has a photometric redshift $0.44 \pm 0.15$, well separated from GRB 130702A.

Table 1

Stellar Masses and Metallicities of Galaxies

\begin{tabular}{lccc}
\hline \hline Name & Separation from Transient & $\begin{array}{c}\text { Mass } \\
\log \left(\mathrm{M} / \mathrm{M}_{\odot}\right)\end{array}$ & $\begin{array}{c}\text { Metallicity } \\
12+\log (\mathrm{O} / \mathrm{H})\end{array}$ \\
\hline S1 & $0.6^{\prime \prime}(1.5 \mathrm{kpc}$ projected on sky) & $7.9 \pm 0.7$ & $\lesssim 8.16$ \\
S2 & $7.6^{\prime \prime}(19.1 \mathrm{kpc}$ projected on sky $)$ & $10.8 \pm 0.1$ & \\
S3 & $15.8^{\prime \prime}(37.8 \mathrm{kpc}$ projected on sky) & $9.5 \pm 0.08^{\mathrm{a}}$ & \\
\hline
\end{tabular}

Note. - Offsets of galaxies from the GRB coordinates, their stellar masses fit from SDSS ugriz galaxy photometry corrected for Milky Way extinction, and oxygen abundance upper limit for source S1.

${ }^{a}$ Only photometric redshift available. We assume that the source has $z=$ 0.145 for the stellar mass estimate.

The standard deviation of the velocities of these bright galaxies is $366 \mathrm{~km} \mathrm{~s}^{-1}$, suggesting that the LGRB host galaxy is a member of a galaxy group with $\sim 2 \mathrm{Mpc}$ size.

From the root-mean-square variation of the continuum and the size of the extraction window for dwarf host S1, we place a $2 \sigma$ upper limit on the [N II] flux of $5.4 \times 10^{-18}$ ergs $\mathrm{cm}^{-2} \mathrm{~s}^{-1}$, while we find an $\mathrm{H} \alpha$ flux of $111 \times 10^{-18}$ ergs $\mathrm{cm}^{-2} \mathrm{~s}^{-1}$. These values allow us to place an upper limit on the Pettini \& Pagel (2004) $[\mathrm{N} \mathrm{II}] / \mathrm{H} \alpha$ abundance of $12+\log (\mathrm{O} / \mathrm{H}) \lesssim 8.16$ dex.

From the $\mathrm{H} \alpha=111 \pm 0.5 \times 10^{-18} \mathrm{ergs} \mathrm{cm}^{-2} \mathrm{~s}^{-1}$ and $\mathrm{H} \beta=43 \pm 5.2 \times 10^{-18}$ ergs $\mathrm{cm}^{-2} \mathrm{~s}^{-1}$ fluxes we measure for the dwarf host galaxy S1, we estimate a Balmer decrement of $\mathrm{H} \alpha / \mathrm{H} \beta=2.6 \pm 0.3$. This value is consistent with the expectation for Case $\mathrm{B}$ recombination (i.e. $\mathrm{H} \alpha / \mathrm{H} \beta=2.86$; Osterbrock 1989) and suggests insignificant reddening. We therefore adopt $A_{V}=0 \mathrm{mag}$ for the emission-line analysis.

Applying the Kennicutt (1998) relation between $\mathrm{H} \alpha$ flux and star-formation rate (SFR), we estimate an SFR of $0.05 \mathrm{M}_{\odot} \mathrm{yr}^{-1}$ for $\mathrm{S} 1$ within the $1^{\prime \prime}$ slit aperture. For comparison, the SFR of the Large Magellanic Cloud is $\sim 0.2 \mathrm{M}_{\odot} \mathrm{yr}^{-1}$ (Harris \& Zaritsky 2009), while the underlying SFR of the Small Magellanic Cloud, excepting intermittent episodes of stronger star formation, has been $\sim 0.1 \mathrm{M}_{\odot} \mathrm{yr}^{-1}$ (Harris \& Zaritsky 2004). The $10^{7.9 \pm 0.7} \mathrm{M}_{\odot}$ stellar mass of $\mathrm{S} 1$ is in the 26th percentile of the host masses of 34 LGRBs measured by Svensson et al. (2010). The galaxy SFR is in the 6th percentile of the SFRs of the LGRB host sample, although the Svensson et al. (2010) estimates rely instead on the rest-frame $U$-band luminosity which may have a systematic offset from the Kennicutt (1998) calibration of $\mathrm{H} \alpha$ flux. 


\section{DISCUSSION AND CONCLUSIONS}

We have found evidence that the host of GRB 130702A is a dwarf galaxy that is a satellite of a nearby massive, metal-rich galaxy. Modeling of the two galaxies' photometry suggests that the dwarf galaxy has a stellar mass $\sim 1 \%$ that of the red, bright galaxy. If we assume that the massive red galaxy consists of a very thin exponential disk, and we project the LGRB coordinates onto the disk's inclined plane, the transient has an offset from the galaxy center of $\sim 60 \mathrm{kpc}$, or $\sim 9$ times the half-light radius. A thick galaxy disk or a significant bulge would imply a greater disk inclination angle and a more remote explosion site. A large central offset suggests that the faint source is a dwarf galaxy and not a part of the massive galaxy's disk.

Comparison between the spectroscopic redshifts of the dwarf and the massive red galaxy shows that the velocity offset between the two galaxies is less than $\sim 60 \mathrm{~km} \mathrm{~s}^{-1}$, which is small in comparison to the $\sim 366 \mathrm{~km} \mathrm{~s}^{-1}$ velocity dispersion measured among five bright galaxies within $\sim 2 \mathrm{Mpc}$ (in projection) of the GRB position having SDSS spectroscopic redshifts.

We find that the massive red primary galaxy likely dominates the local gravitational potential. A blue galaxy is the only bright source that is close in projection, but it would have only $\sim 10 \%$ the stellar mass of the red galaxy if it has $z=0.145$. While these observations offer strong evidence that the dwarf host galaxy is a orbiting satellite of (or merging with) the massive red galaxy, we cannot exclude the possibility of a random but improbable coincidence of both their spatial positions and redshifts.

Inspection of images of the field shows a large number of nearby faint sources, many of whose photometric redshifts are consistent with the GRB spectroscopic redshift. A densely populated environment suggests that group members experience local interactions.

Early comparisons between the host environments of nearby LGRBs and star-forming galaxies suggested that LGRBs strongly prefer metal-poor galaxies (Stanek et al. 2006; Modjaz et al. 2008), but more recent discoveries and analyses have found growing numbers of LGRBs that may have metal-rich host galaxies. Prochaska et al. (2007), for example, used damped Lyman- $\alpha$ (DLA) systems to study the properties of the gas along the line of sight to the explosions at the same redshift as the GRB. They found evidence for metallicities of the gas from $0.01 Z_{\odot}$ to $Z_{\odot}$. Levesque et al. (2010) found that the dark burst GRB 020819B was spatially coincident with a high-metallicity $z=0.41$ galaxy. Recently, Savaglio et al. (2012) inferred supersolar metallicities from zinc and sulfur absorption features for two $z \approx 3.67$ galaxies found in the afterglow spectrum of an LGRB.

This analysis of the host galaxy of GRB 130702A provides the first example of an LGRB that exploded in a dwarf, metal-poor satellite of a massive, metalrich galaxy. In this case, the dwarf galaxy's projection onto the sky from our perspective on Earth placed it away from the primary galaxy. For other examples of LGRBs that explode in dwarf satellite galaxies, however, the dwarf galaxy may be superimposed on a metal-rich galaxy. This suggests the need to obtain a spectrum at the explosion site of the LGRB to attempt to detect the nebular emission of metal-poor gas, as well as highresolution follow-up imaging.

Comparison between the host galaxies of low-redshift SNe Ic-BL without detected GRB emission and normal SNe Ic whose features are not broad finds that the SNe Ic-BL erupt in comparatively metal-poor galaxies (Kelly \& Kirshner 2012; Sanders et al. 2012; see also Arcavi et al. 2010). This pattern at low redshifts where host galaxies are more accessible suggests that low progenitor metallicity may be important to generate explosions with high ejecta velocities. When possible, careful investigation of the immediate environments of LGRBs associated with massive galaxies should help to establish how important metallicity is to the production of gamma-ray emission.

We are very grateful to Brad Cenko for his help in scheduling the observations and reducing the DEIMOS spectra. We also thank Maryam Modjaz for her useful instructions for measuring emission-line fluxes, as well as Daniel Kocevski for discussions about the host-galaxy properties of LGRBs. A.V.F.'s group at UC Berkeley has received generous financial assistance from the Christopher R. Redlich Fund, the TABASGO Foundation, NSF grant AST-1211916, and NASA/Swift grant NNX12AD73G. Some of the data presented herein were obtained at the W. M. Keck Observatory, which is operated as a scientific partnership among the California Institute of Technology, the University of California, and NASA; the observatory was made possible by the generous financial support of the W. M. Keck Foundation.

\section{REFERENCES}

Ahn, C. P., Alexandroff, R., Allende Prieto, C., et al. 2012, ApJS, 203, 21

Arcavi, I., Gal-Yam, A., Kasliwal, M. M., et al. 2010, ApJ, 721, 777

Arnouts, S., Cristiani, S., Moscardini, L., et al. 1999, MNRAS, 310,540

Butler, N., et al. 2013a, GRB Coordinates Network, 14980, 1 —. 2013b, GRB Coordinates Network, 14993, 1

Cenko, S. B., et al. 2013, GRB Coordinates Network, 14998, 1 Cheung, T., et al. 2013, GRB Coordinates Network, 14971, 1 Collazzi, A., et al. 2013, GRB Coordinates Network, 14972, 1 D'Elia, V., et al. 2013, GRB Coordinates Network, 15000, 1

Faber, S. M., Phillips, A. C., Kibrick, R. I., et al. 2003, in Society of Photo-Optical Instrumentation Engineers (SPIE) Conference Series, ed. M. Iye \& A. F. M. Moorwood, Vol. 4841, 1657

Fioc, M., \& Rocca-Volmerange, B. 1999, ArXiv e-prints

Fryer, C. L., \& Heger, A. 2005, ApJ, 623, 302

Galama, T. J., Vreeswijk, P. M., van Paradijs, J., et al. 1998, Nature, 395, 670

Graham, J. F., \& Fruchter, A. S. 2012, ArXiv e-prints, arXiv: 1211.7068

Harris, J., \& Zaritsky, D. 2004, AJ, 127, 1531

-. 2009, AJ, 138, 1243

Hirschi, R., Meynet, G., \& Maeder, A. 2005, A\&A, 443, 581

Hjorth, J., Sollerman, J., Møller, P., et al. 2003, Nature, 423, 847

Kelly, P. L., \& Kirshner, R. P. 2012, ApJ, 759, 107

Kelson, D. D. 2003, PASP, 115, 688

Kennicutt, Jr., R. C. 1998, ApJ, 498, 541

Kocevski, D., \& West, A. A. 2011, ApJ, 735, L8

Kudritzki, R., \& Puls, J. 2000, ARA\&A, 38, 613

Leloudas, G., et al. 2013, GRB Coordinates Network, 14983, 1

Levesque, E. M., Kewley, L. J., Graham, J. F., \& Fruchter, A. S. 2010, ApJ, 712, L26 
Matheson, T., Garnavich, P. M., Stanek, K. Z., et al. 2003, ApJ, 599,394

Modjaz, M., Kewley, L., Kirshner, R. P., et al. 2008, AJ, 135, 1136

Mulchaey, J., et al. 2013, GRB Coordinates Network, 14985, 1

Oke, J. B., \& Gunn, J. E. 1983, ApJ, 266, 713

Osterbrock, D. E. 1989, Astrophysics of Gaseous Nebulae and Active Galactic Nuclei, ed. Osterbrock, D. E.

Pérez-Montero, E., \& Díaz, A. I. 2003, MNRAS, 346, 105

Perley, D., Kasliwal, M., et al. 2013, GRB Coordinates Network, 14979, 1

Pettini, M., \& Pagel, B. E. J. 2004, MNRAS, 348, L59

Prochaska, J. X., Chen, H.-W., Dessauges-Zavadsky, M., \& Bloom, J. S. 2007, ApJ, 666, 267

Rana, N. C., \& Basu, S. 1992, A\&A, 265, 499

Sana, H., de Mink, S. E., de Koter, A., et al. 2012, Science, 337, 444

Sanders, N. E., Soderberg, A. M., Levesque, E. M., et al. 2012, ApJ, 758, 132
Savaglio, S., Rau, A., Greiner, J., et al. 2012, MNRAS, 420, 627 Schlafly, E. F., \& Finkbeiner, D. P. 2011, ApJ, 737, 103

Schlegel, D. J., Finkbeiner, D. P., \& Davis, M. 1998, ApJ, 500, 525

Schulze, S., et al. 2013, GRB Coordinates Network, 14994, 1

Singer, L., et al. 2013, GRB Coordinates Network, 14967, 1

Stanek, K. Z., Matheson, T., Garnavich, P. M., et al. 2003, ApJ, 591, L17

Stanek, K. Z., Gnedin, O. Y., Beacom, J. F., et al. 2006, Acta Astronomica, 56, 333

Svensson, K. M., Levan, A. J., Tanvir, N. R., Fruchter, A. S., \& Strolger, L.-G. 2010, MNRAS, 405, 57

Vink, J. S., \& de Koter, A. 2005, A\&A, 442, 587

Woosley, S. E., \& Heger, A. 2006, ApJ, 637, 914

Woosley, S. E., Langer, N., \& Weaver, T. A. 1993, ApJ, 411, 823

Yoon, S., \& Langer, N. 2005, A\&A, 443, 643

Zhang, W., \& Fryer, C. L. 2001, ApJ, 550, 357 